\title{
Research on Platycodon Grandiflorum Eriobotrya Japonica Honey Tablet Technology and Determination of Content of Platycodin D, Ursolic and Oleanolic Acid
}

\author{
Xiuying Wang ${ }^{1}$ Wei Zhang ${ }^{2}$ Chunlin Zhao ${ }^{1}$ Yang Chen $^{1}$ \\ ${ }^{1}$ Jilin Agricultural Science and Technology University, College of Traditional Chinese Medicine, Jilin, \\ Jilin, China, 132101 \\ ${ }^{2}$ Changchun Beihua Pharmaceutical Co., Ltd, Jilin, Jilin, China, 132101
}

Keywords: Platycodon Grandiflorum, Eriobotrya Japonica, Honey Tablet, Technology

\begin{abstract}
Optimization of honey tablet of Platycodon grandiflorum and Eriobotrya japonica technology was preferred by using the orthogonal experiment which were performed by honey processing time, loquat concentration, soaked temperature as main factors. The superior method was adopted with experiment data with testing honey-tablet yield of Platycodon grandiflorum and Eriobotrya japonica, color, transparency, taste, texture, viscosity and determining content of platycodin D, ursolic and oleanolic acid by HPLC. The results showed that $0.50 \%$ of loquat concentration, $80{ }^{\circ} \mathrm{C}$ of soaked temperature and $85 \mathrm{~min}$ of suitable soaked time were the best preparation technology for honey tablet in which $44.96 \%$ yield of honey tablet and 6 points of total score was were obtained, and $1.323 \mathrm{mg} . \mathrm{g}^{-1}$ content of platycodin $\mathrm{D}, 2.983 \mathrm{mg} \cdot \mathrm{g}^{-1}$ of ursolic acid and $1.068 \mathrm{mg}^{-1} \mathrm{~g}^{-1}$ of oleanolic acid were detected by HPLC.
\end{abstract}

\section{Introduction}

Campanulaceae is also known as alias bell flower, monk cap, lanterns, white medicine, ginseng, Doraji (Korea, Korea) and Kikyo (Japan), etc., for the genus Platycodon grandiflorum (Jacq. Open Xuan lung, expectorant abscess. Modern pharmacology has expectorant, anti-inflammatory, immune regulation, anti-tumor, liver protection, cardiovascular protection, treatment of diabetes, anti-obesity, analgesic and other effects [2]. Loquat leaves for the rosewood loquat Eriobotrya japonica (Thunb.) Lindl. "Doctors do not record" as the goods. Loquat leaves with Qingfei and stomach, lowering gas and phlegm function, is the treatment of Hyperactivity cough and asthma of the drug $[3,4]$, modern pharmacological studies with anti-cancer, anti-tumor and anti-leukemia and other effects $[5,6]$. Honey sweet taste, willing to spleen, lungs cough, taste and so on. Honey Sunburn method for cough and asthma, Spleen Qi drugs [7, 8].

Campanulaceae loquat honey in the traditional Campanulaceae loquat cough syrup compatibility on the basis of research and development of a food and medicine with the use of health food, the use of both mutual help function, the health of the human body function and adjustment, enhance immune function, play Xuanfei phlegm, Asthma cough, qi and soothe the nerves, physical fitness, longevity purposes.

\section{Materials, Apparatus and Reagents}

Test Materials. Campanulaceae (Jilin Agricultural Science and Technology College Plantation 3 years old), loquat leaves (Jilin Jisheng Tong large pharmacy purchased), honey (Antu County Animal Husbandry Bureau Beekeeping provided). The platycodon root to remove impurities washed, cut into $2 \sim 4 \mathrm{~mm}$ thick tablets spare.

Instruments and Reagents. JYT-2 type drug balance (Shanghai Medical Laser Instrument Factory); AL-204 type electronic balance (METTLER TOLEDO Co., Ltd. - Shanghai); WMZK-72 type pointer thermostatic water bath (Shanghai Yuejin Medical Equipment Factory) ; SY-360-type ultrasonic extractor (Shanghai Ning Shang Tong Sheng Instrument Co., Ltd.); YXQ-LS-50S vertical steam sterilizer (Shanghai-source-long experimental equipment factory) (Pure), acetic acid, 
acetonitrile (chromatographic purity, Beijing), glacial acetic acid, triethylamine; reference control of Campanulaceae D, Saponin Acid reference substance, oleanolic acid reference substance, purchased from the China Food and Drug Research Institute.

\section{Test Method}

Preparation Technology of Campanulaceae Loquat Honey. Linden tree honey pot heated to slowly boiling, slow fire slightly boiling, remove the foam, floating wax, dead bees and impurities, heated to $116{ }^{\circ} \mathrm{C}-118{ }^{\circ} \mathrm{C}$, from the fish eye bubble, hand twist of the sticky, between the two fingers without long white Silk, quickly pan. Water content control in $10 \%-13 \%$ is appropriate [8].

The main technological factors of the flavors of Campanulaceae were A (hot-dip temperature 65,80,95 ${ }^{\circ} \mathrm{C}$ ), B (hot-dip time), and the ratio of the extract to honey was mixed with ethanol. : 45, $60,85 \mathrm{~min}$ ), C (loquat concentration $0.25,0.50,1.00 \%$ ), select the orthogonal test L9 (34) factor the level of optimization test design table (see Table 1), so the Campanulaceae loquat prepared Process optimization, final drying weighing calculation yield and quality evaluation.

Table 1 Orthogonal test L9 (34)

\begin{tabular}{ccccc}
\hline Serial number & $\begin{array}{c}\mathrm{A} \\
\text { Boiling } \\
\text { temperature } \\
\left({ }^{\circ} \mathrm{C}\right)\end{array}$ & $\begin{array}{c}\mathrm{B} \\
\text { Boiling time } \\
(\mathrm{min})\end{array}$ & $\begin{array}{c}\mathrm{C} \\
\text { Loquat } \\
\text { concentration } \\
(\%)\end{array}$ \\
\hline 1 & $\mathrm{~A}_{1} \mathrm{~B}_{1} \mathrm{C}_{1}$ & 65 & 45 & 0.25 \\
2 & $\mathrm{~A}_{1} \mathrm{~B}_{2} \mathrm{C}_{2}$ & 65 & 60 & 0.50 \\
3 & $\mathrm{~A}_{1} \mathrm{~B}_{3} \mathrm{C}_{3}$ & 65 & 85 & 1.00 \\
4 & $\mathrm{~A}_{2} \mathrm{~B}_{2} \mathrm{C}_{1}$ & 80 & 60 & 0.25 \\
5 & $\mathrm{~A}_{2} \mathrm{~B}_{3} \mathrm{C}_{2}$ & 80 & 85 & 0.50 \\
6 & $\mathrm{~A}_{2} \mathrm{~B}_{1} \mathrm{C}_{3}$ & 80 & 45 & 1.00 \\
7 & $\mathrm{~A}_{3} \mathrm{~B}_{3} \mathrm{C}_{1}$ & 95 & 85 & 0.25 \\
8 & $\mathrm{~A}_{3} \mathrm{~B}_{1} \mathrm{C}_{2}$ & 95 & 45 & 0.50 \\
9 & $\mathrm{~A}_{3} \mathrm{~B}_{2} \mathrm{C}_{3}$ & 95 & 60 & 1.00 \\
\hline
\end{tabular}

Evaluation of Comprehensive Index of Campanulaceae Loquat Honey. The harvest rate, color, transparency, texture and viscosity of 9 layers of Campanulaceae were investigated and the scoring mechanism was established. Sweetness: sweet fragrance 1.0 points, slightly sweet 0.8 points, slightly bitter 0.6 points; viscosity: slightly sticky 1.0 points, stick 0.8 points, not sticky 0.6 points; smell: fragrance 1.0 points, fragrance 0.8 points, Color: yellow 1.0, light yellow 0.8, brown 0.6 ; texture: soft and hard moderate 1.0 points, soft 0.8 , hard 0.6 points [10].

D Detection of Campanulaceae Saponin. The mobile phase consisted of acetonitrile-water (26:74), flow volume $1.0 \mathrm{ml} / \mathrm{min}$, injection volume $10 \mu \mathrm{l}$, detection wavelength $472 \mathrm{~nm}$, column temperature $20{ }^{\circ} \mathrm{C}$.

$(100 \mathrm{~W}, 50 \mathrm{~Hz}$ ), after taking the liquid liquid $25 \mathrm{ml}$, fully dried, the volume of $10 \mathrm{ml}$ of methanol will be prepared after the dry powder, dried and then ground into a fine powder, weighed $2.0 \mathrm{~g}, 50 \%$ methanol 50ml, ultrasonic extraction $30 \mathrm{~min}(100 \mathrm{~W}, 50 \mathrm{~Hz})$

Take $0.1,0.2,0.5,1.0,2.0,4.0 \mathrm{ml}$ to $10 \mathrm{ml}$ volumetric flask, according to the above conditions, the injection volume of $10 \mu \mathrm{l}$, the peak area is divided into the ordinate, Campanulaceae $\mathrm{S}$ is the abscissa plot standard curve, The regression equation $\mathrm{Y}=1.233 \mathrm{X}+13.443, \mathrm{r}=0.9996$, Campanulaceae D in $0.025-0.100 \mathrm{mg} / \mathrm{ml}$ showed a good linear relationship.

Determination of Ursolic Acid and Oleanolic Acid [11]. (90: 10: 0.03: 0.06) as the mobile phase, the volume flow rate was $1.0 \mathrm{ml} / \mathrm{min}$, the injection volume was $10 \mu 1$, and the concentration of the sample was measured. The wavelength is $205 \mathrm{~nm}$ and the column temperature is $20{ }^{\circ} \mathrm{C}$.

$(100 \mathrm{~W}, 50 \mathrm{~Hz}$ ), filtered $25 \mathrm{ml}$ after the liquid, fully dried, add $10 \mathrm{ml}$ of the volume of methanol to 
prepare. (2), 50ml methanol 50ml, ultrasonic extraction 30min (100W, 50Hz)

Respectively, take $0.1,0.2,0.5,1.0,2.0,4.0 \mathrm{ml}$ to $10 \mathrm{ml}$ volumetric flask volume, according to the above conditions, the injection rate of $10 \mu \mathrm{l}$, the peak area is divided into the ordinate, ursolic acid and oleanolic acid peak The area is plotted as the abscissa of the standard curve, the regression equation, ursolic acid $\mathrm{Y}=0.0056 \mathrm{X}+0.0359, \mathrm{r}=0.9993$; oleanolic acid $\mathrm{Y}=0.0044 \mathrm{X}-0.0122, \mathrm{r}=$ 0.9995. The linear ranges were 0.030 to $0.0120 \mathrm{mg} / \mathrm{ml}$ and $0.031 \mathrm{~L}$ to $0.0124 \mathrm{mg} / \mathrm{ml}$, respectively.

\section{Results and Analysis}

Analysis on the Comprehensive Index of Yield and Appearance of Campanulaceae Loquat. (Yield = W2 / W1 × 100\%; W2 dried after the quality of Campanulaceae wafers; W1 fresh platycodon tablets) quality and appearance of the quality of the evaluation of the quality of the product See Table 2 for details.

Table 2 Comprehensive quality evaluation and yield of Platycodon grandiflorum Eriobotrya japonica honey tablet

\begin{tabular}{llllllllll}
\hline Test number & Yield $(\%)$ & $\begin{array}{l}\text { Taste } \\
\text { Sweetness }\end{array}$ & Viscosity & odor & transparency & Color & texture & $\begin{array}{l}\text { Overall } \\
\text { rating }\end{array}$ \\
\hline 1 & $\mathrm{~A}_{1} \mathrm{~B}_{1} \mathrm{C}_{1}$ & 37.87 & 0.6 & 0.6 & 0.6 & 0.6 & 0.6 & 0.6 & 3.6 \\
2 & $\mathrm{~A}_{1} \mathrm{~B}_{2} \mathrm{C}_{2}$ & 39.31 & 0.8 & 0.6 & 0.6 & 0.8 & 0.8 & 0.6 & 4.2 \\
3 & $\mathrm{~A}_{1} \mathrm{~B}_{3} \mathrm{C}_{3}$ & 41.48 & 1.0 & 1.0 & 1.0 & 0.8 & 0.8 & 0.8 & 5.4 \\
4 & $\mathrm{~A}_{2} \mathrm{~B}_{2} \mathrm{C}_{1}$ & 43.17 & 0.8 & 1.0 & 0.6 & 1.0 & 1.0 & 1.0 & 5.4 \\
5 & $\mathrm{~A}_{2} \mathrm{~B}_{3} \mathrm{C}_{2}$ & 44.96 & 1.0 & 1.0 & 1.0 & 1.0 & 1.0 & 1.0 & 6.0 \\
6 & $\mathrm{~A}_{2} \mathrm{~B}_{1} \mathrm{C}_{3}$ & 39.62 & 0.8 & 0.6 & 0.8 & 0.6 & 0.8 & 0.8 & 4.4 \\
7 & $\mathrm{~A}_{3} \mathrm{~B}_{3} \mathrm{C}_{1}$ & 46.62 & 0.8 & 0.8 & 1.0 & 1.0 & 1.0 & 1.0 & 5.6 \\
8 & $\mathrm{~A}_{3} \mathrm{~B}_{1} \mathrm{C}_{2}$ & 38.65 & 0.8 & 0.6 & 0.8 & 0.8 & 0.6 & 0.8 & 4.4 \\
9 & $\mathrm{~A}_{3} \mathrm{~B}_{2} \mathrm{C}_{3}$ & 45.24 & 1.0 & 1.0 & 1.0 & 1.0 & 0.8 & 1.0 & 5.8 \\
average & 41.87 & 0.84 & 0.8 & 0.82 & 0.84 & 0.82 & 0.84 & 4.98 \\
value & & & & & & & & \\
\hline
\end{tabular}

The results showed that the yield of 4,5,7,9 Campanulaceae loquat was higher, which was $43.17 \%, 44.96 \%, 46.62 \%$ and $45.24 \%$, respectively, which were higher than the average value of $41.87 \%$ The highest yield. The yield of 1, 2, 3, 6, and 8 was lower than the average, and the yield of treatment 1 was the lowest, which was $37.87 \%$. The results showed that the overall trend of the yield of Campanulaceae loquat was related to the preparation time and temperature of the flax, the high temperature and the long harvest rate were higher, which may increase with time and temperature, more, so that the weight has increased due. The overall quality evaluation scores for treatments 3, 4, 5, 7, 9 were 5.4, 5.4, 6.0, 5.6 and 5.8, respectively, where treatments 5, 7 and 9 were optimal, and 9 loquat concentrations were high , Treatment 7 long heating time, high heating temperature and high viscosity affect the taste. In summary, the treatment rate of 5, odor, transparency, color, texture and taste are better, for the best treatment program.

Determination of Platycodin D, Ursolic Acid and Oleanolic Acid in Platycodon grandiflorum by HPLC 


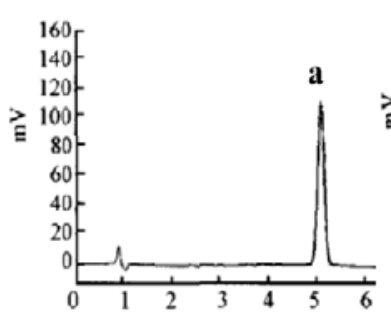

A

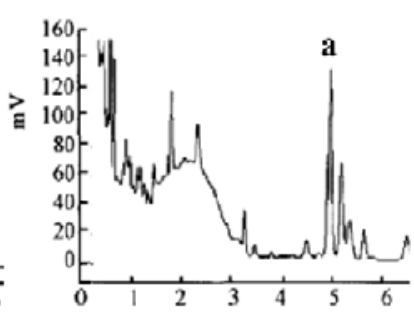

B

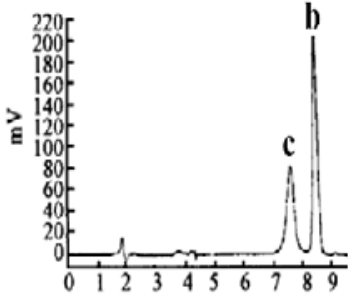

C

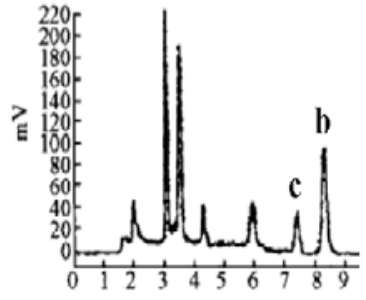

D

Figure 1 a Platycodin D; b ursolic; c oleanolic acid

(A standard; B samble; C standard; D samble)

The content of platycodin D, ursolic acid and oleanolic acid were measured in five treatments with high scores in the yield and overall quality assessment

The three main components (Figure 1) were detected in the samples by HPLC. The contents are shown in Table 3.

Table 3 The content of Platycodin D, ursolic and oleanolic acid of Platycodon grandiflorum Eriobotrya japonica honey tablet

\begin{tabular}{|c|c|c|c|c|c|c|c|c|c|c|c|c|}
\hline \multirow{3}{*}{$\begin{array}{r}\text { Test } \\
\text { number }\end{array}$} & & \multicolumn{3}{|c|}{ Platycodin content（mg.g-1） } & \multicolumn{3}{|c|}{ Ursolic acid content（mg.g-1） } & \multicolumn{5}{|c|}{ Oleanolic acid（mg.g-1） } \\
\hline & & & avera & RSD & & avera & RSD & & conte & avera & & RSD \\
\hline & & & ge value & $\%$ & & & $\%$ & $\mathrm{nt}$ & & ge value & $\%$ & \\
\hline \multirow{3}{*}{$\mathrm{C}_{3}$} & \multirow{3}{*}{$\mathrm{A}_{1} \mathrm{~B}_{3}$} & 1.421 & & & 5.888 & & & & 1.921 & & & \\
\hline & & 1.410 & 1.414 & 0.34 & 5.914 & 5.915 & 2.21 & & 1.945 & 1.924 & & 0.83 \\
\hline & & 1.412 & & & 5.942 & & & & 1.906 & & & \\
\hline \multirow{3}{*}{ ' $\mathrm{C}_{1}$} & \multirow{3}{*}{$\mathrm{A}_{2} \mathrm{~B}_{2}$} & 1.393 & & & 1.412 & & & & 0.512 & & & \\
\hline & & 1.344 & 1.370 & 2.54 & 1.386 & 1.402 & 0.82 & & 0.537 & 0.516 & & 3.13 \\
\hline & & 1.372 & & & 1.408 & & & & 0.498 & & & \\
\hline \multirow{3}{*}{$\mathrm{C}_{2}$} & \multirow{3}{*}{$\mathrm{A}_{2} \mathrm{~B}_{3}$} & 1.304 & & & 2.945 & & & & 1.095 & & & \\
\hline & & 1.322 & 1.323 & 1.20 & 3.014 & 2.982 & 0.99 & & 1.036 & 1.068 & & 2.29 \\
\hline & & 1.343 & & & 2.986 & & & & 1.074 & & & \\
\hline \multirow{3}{*}{$\mathrm{C}_{1}$} & \multirow{3}{*}{$\mathrm{A}_{3} \mathrm{~B}_{3}$} & 1.114 & & & 1.612 & & & & 0.584 & & & \\
\hline & & 1.085 & 1.116 & 2.38 & 1.632 & 1.603 & 1.75 & & 0.536 & 0.566 & & 3.81 \\
\hline & & 1.150 & & & 1.565 & & & & 0.579 & & & \\
\hline \multirow{3}{*}{$\mathrm{C}_{3}$} & \multirow{3}{*}{$A_{3} B_{2}$} & 1.262 & & & 6.771 & & & & 2.145 & & & \\
\hline & & 1.313 & 1.302 & 2.24 & 6.752 & 6.731 & 0.64 & & 2.173 & 2.161 & & 0.54 \\
\hline & & 1.331 & & & 6.671 & & & & 2.164 & & & \\
\hline
\end{tabular}

Table 3 data show that Campanulaceae S D content: treatment 7 minimum, 1.116mg.g-1, treatment 3 the highest, 1.414mg.g-1, which is heating time and heating temperature, with the heating temperature, Time lengthened, Campanulaceae saponin dissolved in honey, resulting in decreased content of honey. Analysis of the visual diagram (Figure 2) shows that the content of ursolic acid and oleanolic acid is related to the concentration of loquat extract. The content of ursolic acid in treatments 3 and 9 was $5.915 \mathrm{mg} . g-1$ and $6.731 \mathrm{mg} . g-1$, respectively, and the difference was significantly different from that of Campanulaceae $S$, which was more than 3 times that of Campanulaceae S, and may be related to the addition of loquat extract concentration (1\%); the oleanolic acid concentrations of treatments 4 and 7 were low, and their contents were $37.7 \%$ and $50.7 \%$, respectively, which may be related to the addition of loquat extract $(0.25 \%)$, while The content of ursolic acid was $0.983 \mathrm{mg}$.g-1, the oleanolic acid contained $1.068 \mathrm{mg}$.g-1, and the content of Campanulaceae S was 1.323mg.g-1 And the concentration of the active ingredients was close to that of the traditional Chinese medicine. The optimum compatibility condition was determined by adding the loquat extract at $0.50 \%$.

\section{Conclusion and Discussion}

Through the comparison of the yield of 9 treatments and the comprehensive quality evaluation score, the treatment of 3, 4, 5, 7, 9 with high yield and comprehensive quality score was selected for the treatment of Campanulaceae D, Ursolic Acid And the content of oleanolic acid were determined. The results of the experimental data show that the scores of treatment 5 (A2B3C2) and 9 (A3B2C3) 
are 6.0 and 5.8, respectively, which are higher than those of 3, 4 and 7, and the yield of post-drying treatment is slightly higher than that of Processing 5. In accordance with the requirements of the process, these two processes have reached the ideal level of technology. Treatment of 5 Campanulaceae Saponin D content slightly higher than the treatment 9, and treatment 9 ursolic acid (Trichoderma saponins D content of more than 3 times), oleanolic acid content is too high, with the same treatment of Campanulaceae saponin D content deviation.

Combined with the effective components of traditional Chinese medicine in the content is relatively close to the case, the synergistic effect is strong, the drug efficacy is better to play, comprehensive analysis and evaluation, choose to deal with 5 method of Campanulaceae loquat honey processing technology is the best, the specific process: System time 85min, loquat concentration $0.50 \%$, immersion temperature $80{ }^{\circ} \mathrm{C}$.

The content of Campanulaceae was $1.323 \mathrm{mg} . g-1$, the content of ursolic acid was $2.983 \mathrm{mg} . \mathrm{g}-1$, the content of oleanolic acid was $1.068 \mathrm{mg} . \mathrm{g}-1$, which was lower than that of loquat Platycodon granules and loquat cough syrup content. Campanulaceae loquat honey is the development of functional health food, not to cure the main purpose is to live on the basis of diet play a role in health care for human health escort, so the active ingredient content is low.

\section{References}

[1] Guo Li, Zhang Cun, Li Li, Xiao Yongqing. Chinese medicine Campanulaceae research progress [J]. Chinese Journal of Traditional Chinese Medicine, 2007, (32) 3: $181 \sim 184$

[2] Li Ting, Xu Wenshan, Li Xiwen, Chen Xiuping, Lu Jinjian. Chinese medicine Campanulaceae modern pharmacological research progress [J]. Chinese Journal of Pharmacology and Clinical, 2013,2: $205 \sim 208$

[3] Lin Yulin, Lin Wenjin, Lin Liqiang. Research status and development prospect of loquat leaves [J]. Chinese Traditional Medicine, 2006, 29 (10); $1111 \sim 1113$

[4] Wang Liwei, Liu Xinmin, Yu Shichun et al. Study on anti-inflammatory and antitussive effects of loquat leaves [J]. Chinese Traditional Medicine, 2004,36 (2): $174 \sim 176$

[5] Wang Lixin, Han Guangxuan, Liu Wenyong, Zhang Weidong. Studies on the chemical and pharmacological properties of oleanolic acid[J]. Chinese Journal of Pharmaceutical Practice, 2001, 19 (2): 104 107

[6] Chiang LC, Chiang W, Chang MY, et al. Antileukemic activity of selected natural products in Taiwan [J] .Am J Chin Med, 2003, 31 (1): $37 \sim 46$

[7] Liu Yihua, Mu Xiaoyan. On the method of Chinese medicine honey Sunburn [J]. Chinese Community Physician (Integrated Edition), 2007,155 (9): 7

[8] Zhu Wei, Wang Xuemei .Mixiao Chinese medicine on the [J]. Tianjin Chinese Medicine. Tianjin Chinese Medicine, 2006 (2): 135 137

[9] Gong Qianfeng, Ding Anwei. Chinese medicine processing system. Beijing, China Chinese Medicine Publishing House, 2007.1: 219 220

[10] Xi Xianrong, Chen Qing. Orthogonal design of licorice honey system technology [J]. Chinese Journal of Traditional Chinese Medicine .2001, 26 (7): 14 18

[13] Li Hongyan, Zhang Rui, Feng Xiaochuan. Determination of Platycodin D, Ursolic Acid, Oleanolic Acid and Capricillin in Compound Loquat Cough Granules by HPLC-ELSD Method[J]. Chinese Traditional Patent Medicine, 2013, 35 (7) : 1479 1484 